\title{
Aspectos prácticos en la patología venosa del pene
}

\section{Practical Aspects in Venous Pathology of the Penis}

\author{
Alejandro Fernández Duque ${ }^{1}$ Juan Fernando Uribe-Arcila ${ }^{2}$ \\ ${ }^{1}$ Clínica de la Mujer, Bogotá, Colombia \\ 2 Departamento de Urología, Hospital Pablo Tobón Uribe, Medellín, \\ Colombia \\ Dirección para correspondencia Juan Fernando Uribe Arcila, MD, \\ Carrera 48, no. 19A-40, Consultorio 1412, Torre Médica Ciudad del \\ Río, Medellín, Antioquia, Colombia \\ (e-mail: medicinasexual@une.net.co).
}

Urol Colomb 2021;30(4):e313-e318.

\section{Resumen \\ Palabras Clave \\ - pene \\ - anatomía \\ - venas \\ - malformaciones venosas}

El objetivo del presente artículo es mostrar una serie de alteraciones peneanas que, aunque frecuentes en la consulta de medicina sexual, son minimizadas, la mayoría de las veces por desconocimiento de los terapeutas, aunque para los pacientes que las padecen sean genuinas tragedias personales. Hemos revisado la literatura disponible sobre un grupo de seis patologías venosas especificas del pene que configuran el grupo más representativo. Se incluyen las frecuentes e indolentes venas superficiales tortuosas, los cirsoceles o flebectasias, las fugas venosas dorsales, que suelen ser una patología congénita, las deformantes malformaciones venosas del glande, la desconcertante y enigmática enfermedad de Mondor, y las fístulas arteriovenosas, con su riesgo de priapismo de alto flujo implícito. Todas se consideran alteraciones patológicas que requieren intervenciones menores en su tratamiento, excepto ciertos casos de fugas venosas y de fístulas, cuyo manejo es invasivo, y que, en conjunto, buscan una mejor comprensión de los hallazgos cotidianos en este órgano.

The objective of the present article is to describe a series of penile alterations that, although frequent in the sexual medicine consultation, are minimized, most of the time due to ignorance of the therapists, but, for the patients who suffer them, they are real personal tragedies. We have reviewed the literature available on a group of six specific venous pathologies of the penis that make up the most representative group. They include the frequent and indolent tortuous superficial veins, the cirsoceles or phlebectasias, the dorsal venous leaks, which are usually a congenital pathology, the deforming venous malformations of the glans, the enigmatic Mondor disease, and the arteriovenous fistulas, with their implicit risk of high-flow priapism. All are considered pathological alterations that require minor interventions in their treatment, except for certain cases of venous leaks and fistulas, whose management is invasive, and which together seek a better understanding of the common findings in this organ. recibido

10 de febrero de 2021

aceptado

13 de agosto de 2021
DOI https://doi.org/

10.1055/s-0041-1736596.

ISSN 0120-789X.

e ISSN 2027-0119.
(C) 2021. Sociedad Colombiana de Urología. All rights reserved. This is an open access article published by Thieme under the terms of the Creative Commons Attribution-NonDerivative-NonCommercial-License, permitting copying and reproduction so long as the original work is given appropriate credit. Contents may not be used for commercial purposes, or adapted, remixed, transformed or built upon. (https://creativecommons.org/ licenses/by-nc-nd/4.0/)

Thieme Revinter Publicações Ltda., Rua do Matoso 170, Rio de Janeiro, RJ, CEP 20270-135, Brazil 


\section{Introducción}

El pene es un órgano complejo, en ocasiones poco estudiado en la cátedra médica, a pesar de su importancia en la estructura biológica y psicológica del género masculino, con enormes implicaciones no solo en el aspecto sexual y reproductivo, sino que en las demás esferas vitales. Aunque existan decenas de miles de publicaciones sobre la estructura y función peneana, las disponibles sobre el tema específico de los problemas venosos son escasas e incompletas. En nuestra práctica, tendemos a creer que consultas de este tipo, que son frecuentes, son aparentemente fáciles de resolver, sin importancia, y, por tanto, no son objeto de estudios sistemáticos. ¿Cuántas veces pacientes nos han preguntado por el tema de las venas tortuosas peneanas y no hemos sabido qué proponer? Como ejemplos, se puede resaltar el tema de las fugas venosas dorsales, que se sospecha cada vez más frecuentemente en consulta, en especial con la actualización en los métodos de estudio; las malformaciones vasculares, que son raras, pero en el ejercicio profesional, casos como el de la enfermedad de Mondor, una patología frecuente que no se diagnostica adecuadamente; y las fistulas arteriovenosas. que siempre generan enormes dudas, y en las que la demora puede tener complicaciones. El objetivo del presente artículo es revisar los datos disponibles en la literatura sobre las seis patologías venosas más comunes en la práctica uroandrológica, cuya información se encuentra dispersa en las bases de datos.

\section{Métodos}

Se realizó una revisión de literatura disponible hasta enero de 2021 en las bases de datos de Google y PubMed para identificar artículos que examinaron la patología venosa del pene con los siguientes términos de búsqueda incluidos: venas AND pene, patología venosa peneana, terapias venosas en pene, venas superficiales tortuosas, cirsoceles oflebectasias, fugas venosas dorsales, malformaciones venosas del glande, enfermedad de Mondor, y fístulas arteriovenosas peneanas. Se encontraron inicialmente 1.557 artículos, pero, luego de aplicados los filtros, quedaron 63 artículos para revisión.

\section{Venas Superficiales Tortuosas o Dilatadas}

Las venas superficiales pueden tener una amplia gama de variaciones anatómicas en su trazado, en cualquiera de las topografías corporales, incluyendo el pene, en el que a menudo se muestran tortuosas o engrosadas, con magnificación durante la fase de erección. Como preocupan al paciente por su desmesura y visibilidad, pueden ser una causa de consulta, pero sólo son una variante anatómica normal, sin consecuencias patológicas (-Figuras 1 y 2 ).,

\section{Flebectasias y Cirsoceles}

Las flebectasias o los cirsoceles (del griego kirsos, varices, y kélé, tumor) son lesiones de origen venoso, las cuales consisten en dilataciones, descritas como lagos venosos o várices, que no están asociadas a ninguna patología, y pueden aparecer en el glande, en el surco balánico, o en el cuerpo del

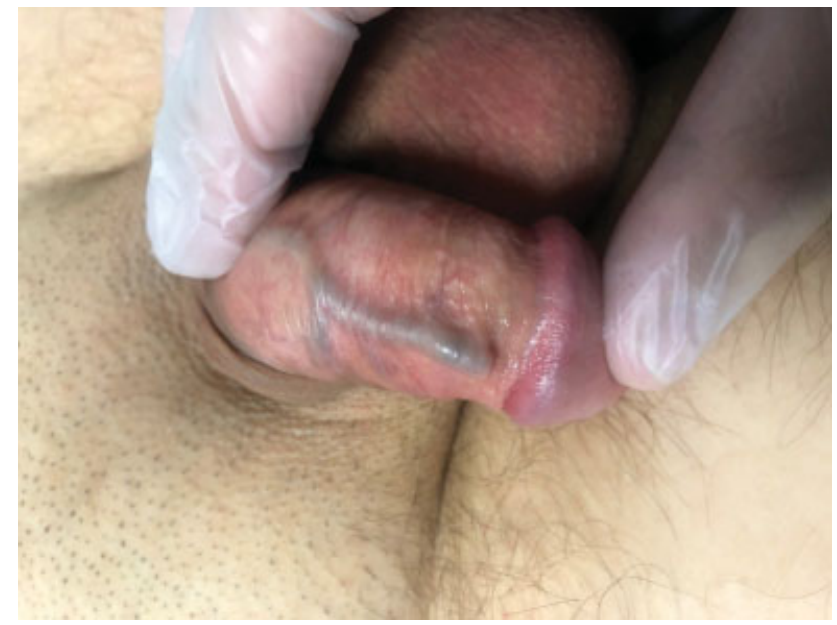

Fig. 1 Vena superficial dilatada en el cuerpo del pene.

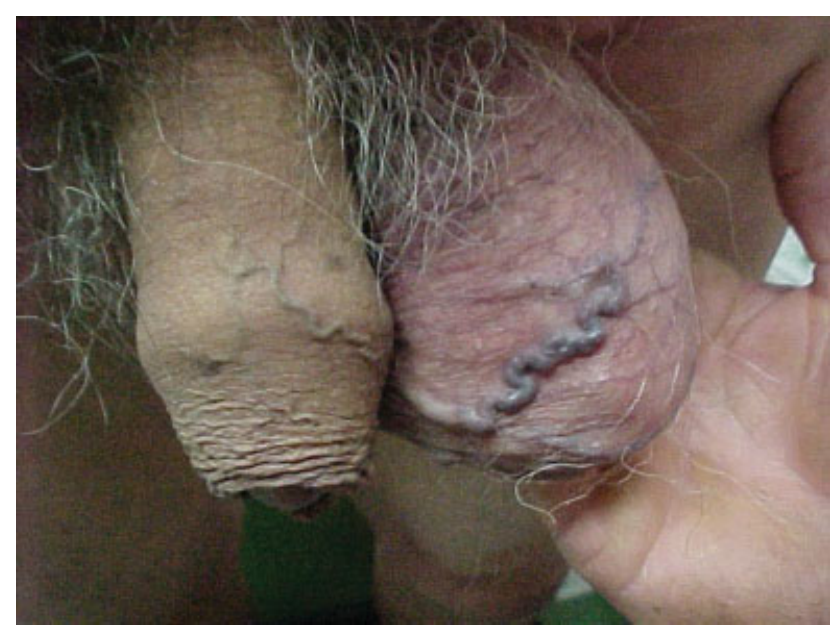

Fig. 2 Venas superficiales tortuosas en el pene y el escroto.

pene; los cirsoceles en el escroto se asocian a la presencia de varicocele, pero esta relación no es tan clara para los del pene. Las lesiones desaparecen con la digito-presión, y reaparecen cuando la presión es liberada; no hay una causa que las explique: pueden ser congénitas, y se ha propuesto que los cambios hormonales y fisiológicos de la pubertad están asociados con su aparición, y algunas veces están relacionadas con el trauma. Las lesiones que algunas veces son más evidentes con la erección pueden causar molestias e incluso dolor intenso para las relaciones sexuales. La ecografía doppler del pene suele mostrar el gran lago venoso, con múltiples señales vasculares y un trazado típico venoso. En general, no es una condición adversa, y rara vez requiere un manejo, excepto cuando implican una molestia sexual, usualmente por dolor. El tratamiento médico puede incluir antiinflamatorios no esteroideos (AINEs), esteroides tópicos, flavonoides con acción flebotónica, y medidas simples como los paños de sulfato de magnesio. El tratamiento quirúrgico se puede realizar con escleroterapia o cirugía de escisión de ser necesario (-Figuras 3,4 y 5). ${ }^{3-7}$ 


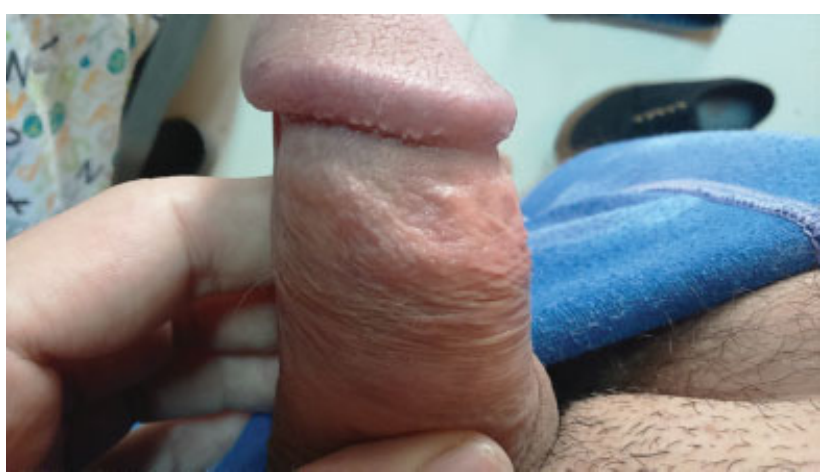

Fig. 3 Flebectasia localizada en el cuerpo del pene.

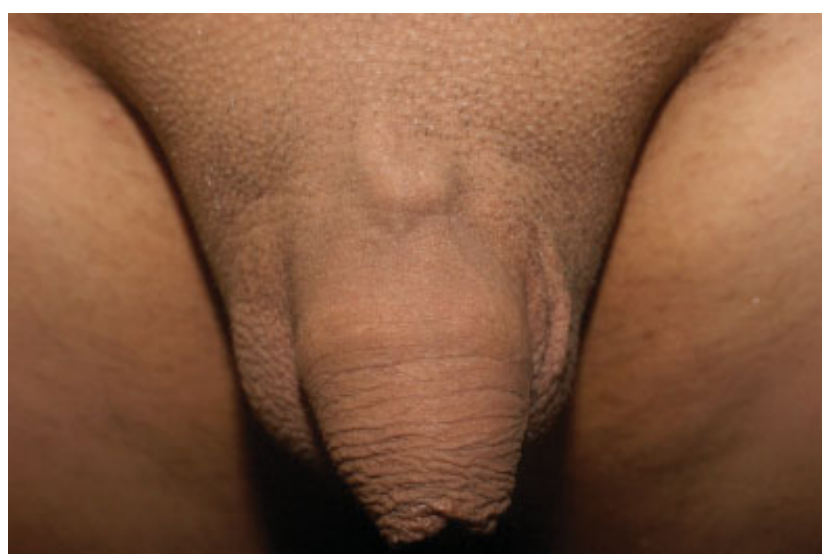

Fig. 4 Flebectasia localizada en la base del pene.

\section{Fugas Venosas Dorsales}

Los mecanismos de la erección humana son una compleja e intrincada red en la que intervienen factores hormonales, neurológicos, arteriales, venosos, y psicológicos. El peso de cada uno de estos elementos es variable; sin embargo, la disfunción venoclusiva (veno-occlusive disease, VOD, por sus siglas en inglés) peneana está siendo reconocida como una causa fundamental de disfunción eréctil en pacientes jóvenes, y se ha encontrado una asociación con el diseño de la ultraestructura del pene, especialmente la arquitectura de la túnica albugínea, que hacen de esta etiología un campo que apenas comienza a estudiarse adecuadamente, con nuevos enfoques de tratamiento disponibles. Los pacientes jóvenes con disfunción eréctil, sobre todo con patrón de perdida fácil de erección, llamado patrón vacilante, ante la disminución de la excitación o concentración, deben ser revisados para un diagnostico posible de fuga venosa congénita. La ecografía doppler de pene es una herramienta económica para la evaluación de la hemodinámica peneana que permite, a su vez, dilucidar el estado del sistema arterial y el comportamiento venoso durante las diferentes fases de la erección, y localizar adecuadamente la fuga venosa dorsal con alguno de los patrones disponibles que usan el número de venas dorsales profundas fugantes, el número de venas superficiales, y las arterias dorsales (- Figuras 6,7 y 8). ${ }^{8-19}$

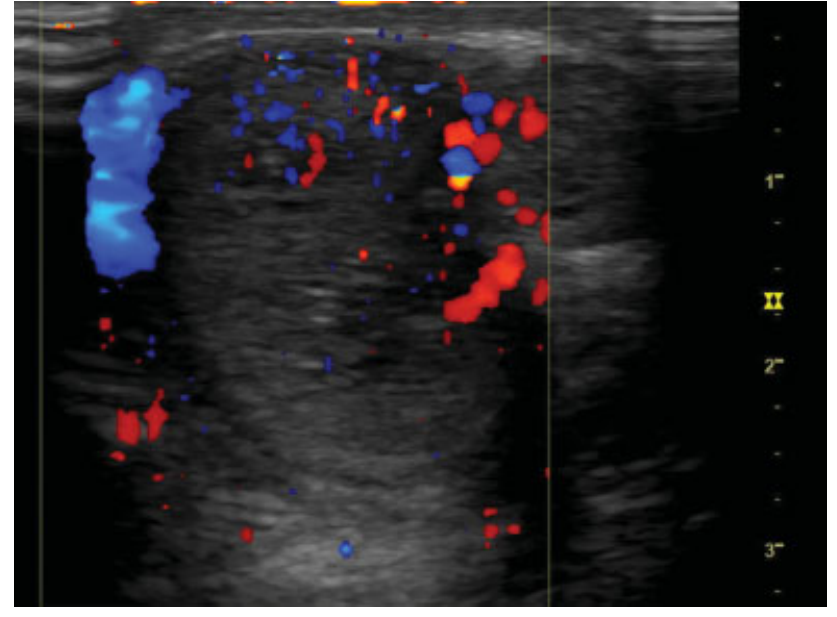

Fig. 5 Flebectasia en ecografía doppler localizada en el cuerpo cavernoso derecho.

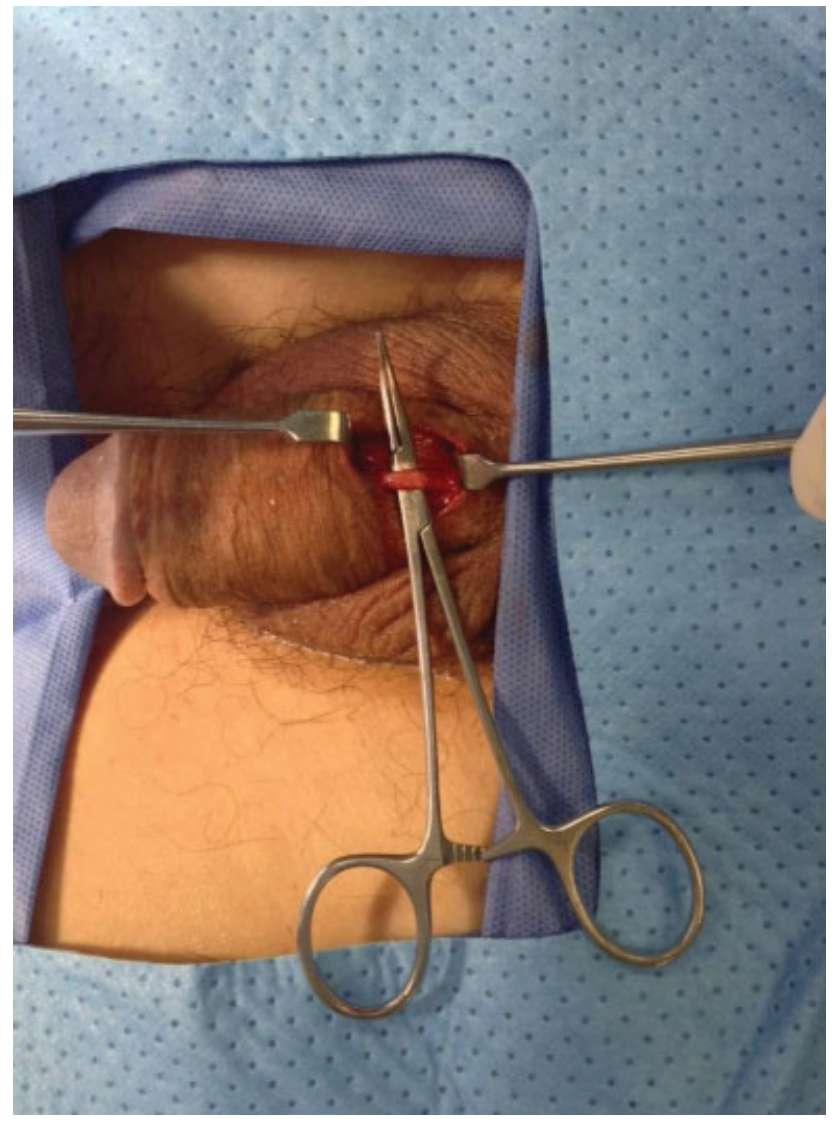

Fig. 6 Vena dorsal durante cirugía de fuga venosa.

\section{Malformaciones Venosas del Glande}

Las malformaciones venosas del glande son raras y tienen una amplia gama de presentaciones. Cuando son severas, suelen ser reconocidas y manejadas desde la infancia, en especial cuando producen una marcada deformidad y compromiso estético. Las modalidades de tratamiento incluyen la escisión quirúrgica, criocirugía, escleroterapia intralesional, y uso de láser (usualmente, granate de itrio y aluminio dopado con impurezas de neodimio, o neodymium- 


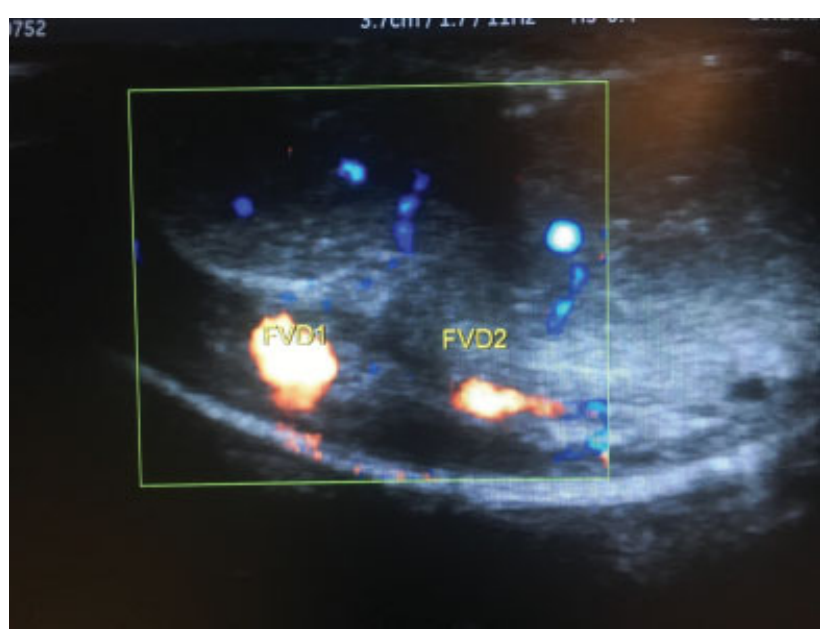

Fig. 7 Dos fugas venosas dorsales profundas en ecografía doppler de pene.

doped yttrium aluminium garnet, Nd:YAG, por sus siglas en inglés) (-Figura 9). ${ }^{20,21}$

\section{Enfermedad de Mondor}

El epónimo enfermedad de Mondor se encuentra referido en la literatura médica a la tromboflebitis superficial (que fue primariamente descrita en venas de la pared torácica), y, luego, adoptado en forma análoga para el mismo fenómeno

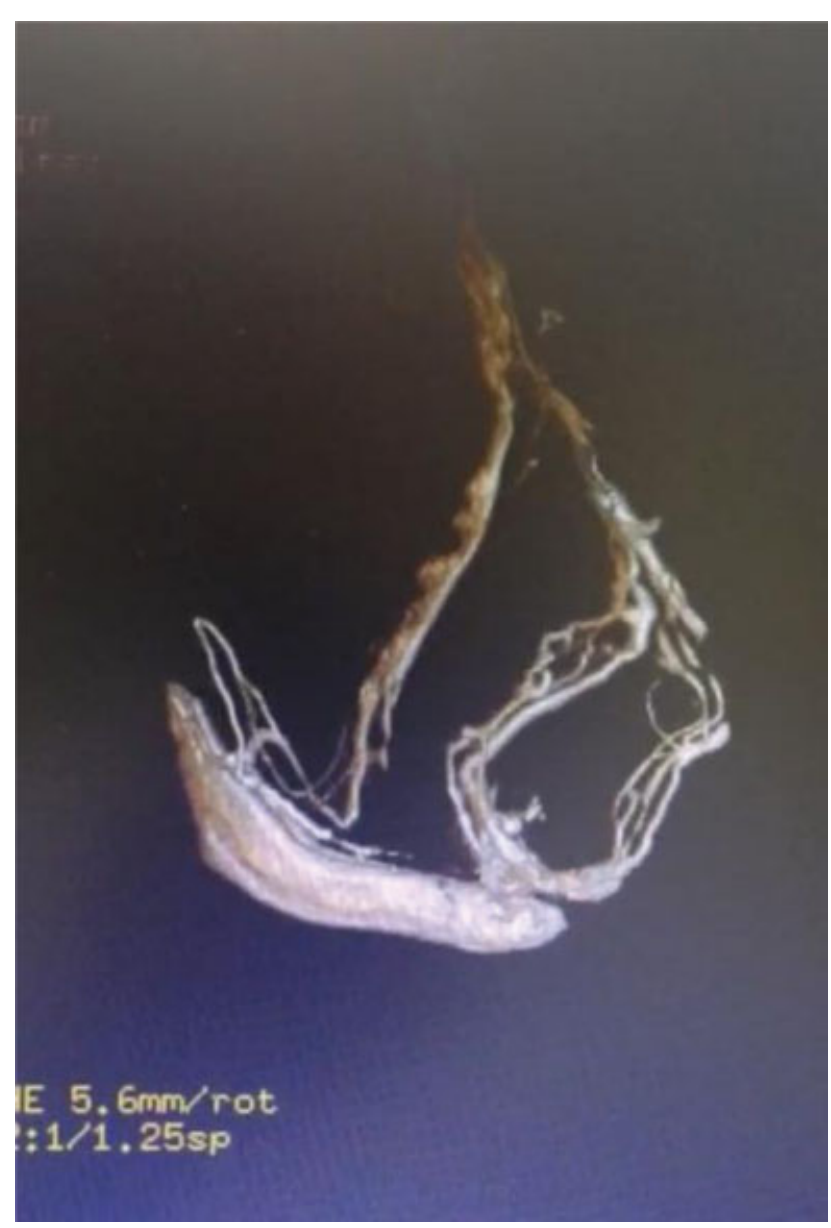

Fig. 8 Dos fugas venosas dorsales profundas en cavernosografía.

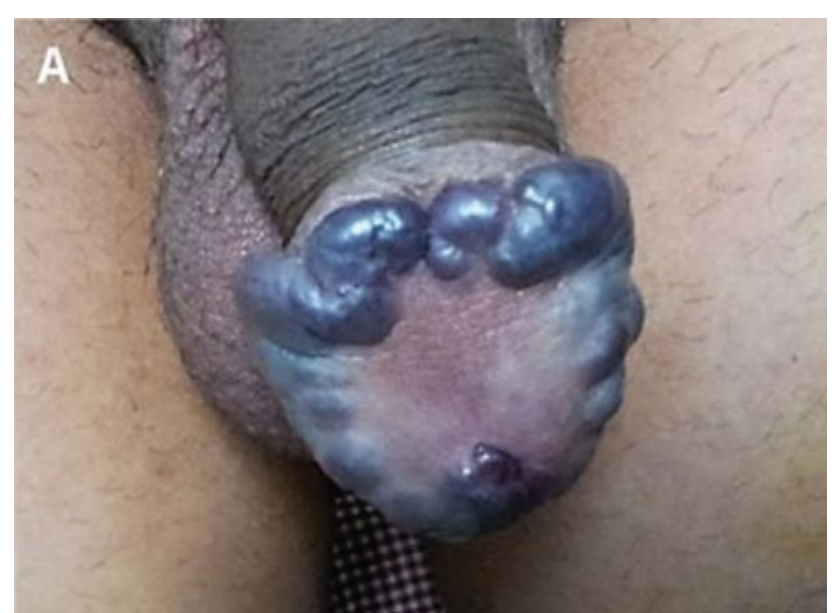

Fig. 9 Malformación venosa del glande peneano (tomada de: Kolte et al. ${ }^{20}$ ).

en las venas superficiales del pene, usualmente una de las venas dorsales superficiales, aunque otras venas pueden ser las afectadas. La descripción se atribuye a Braun-Falco y colaboradores $^{22}$ en 1955 . Se produce un cuadro de dolor e inflamación agudo localizado, que sigue el recorrido de la vena, la mayoría de las veces autolimitado. Los factores predisponentes se encuentran relacionados con situaciones de trauma agudo, microtrauma sexual a repetición, actividad sexual prolongada $\mathrm{y} / \mathrm{o}$ excesiva, abstinencia

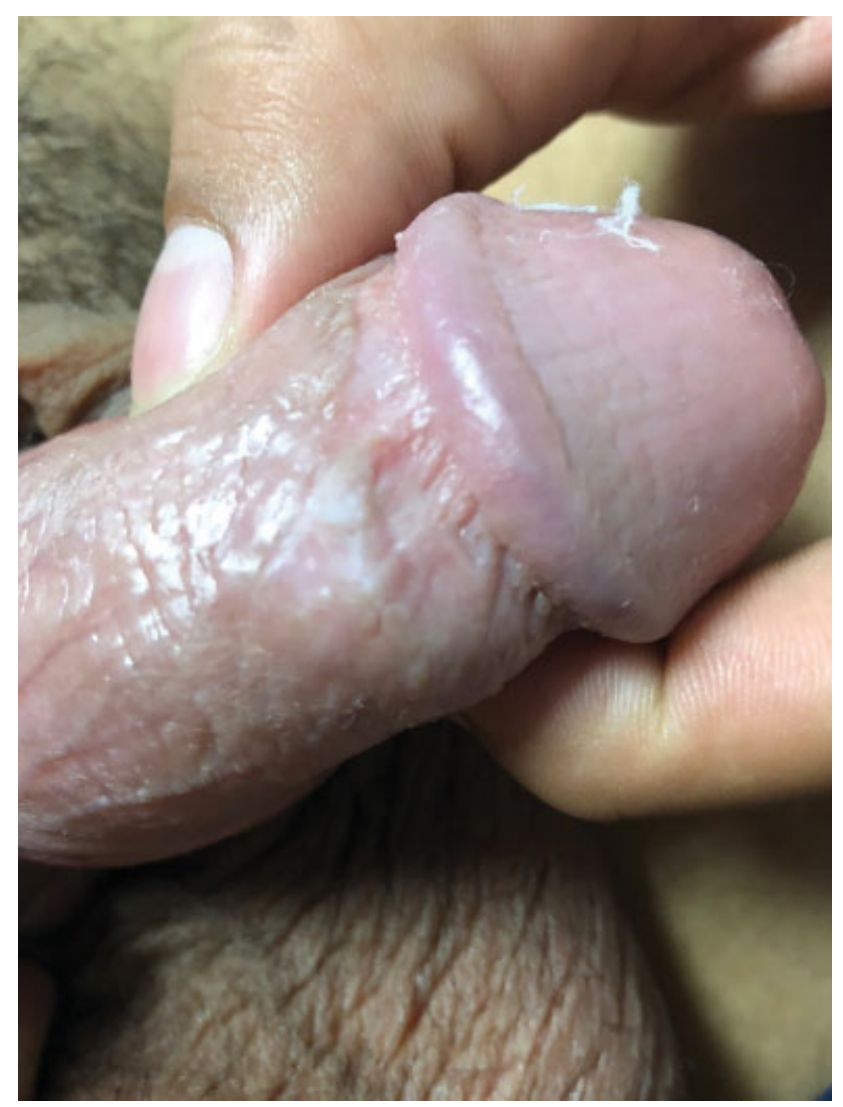

Fig. 10 Enfermedad de Mondor. 


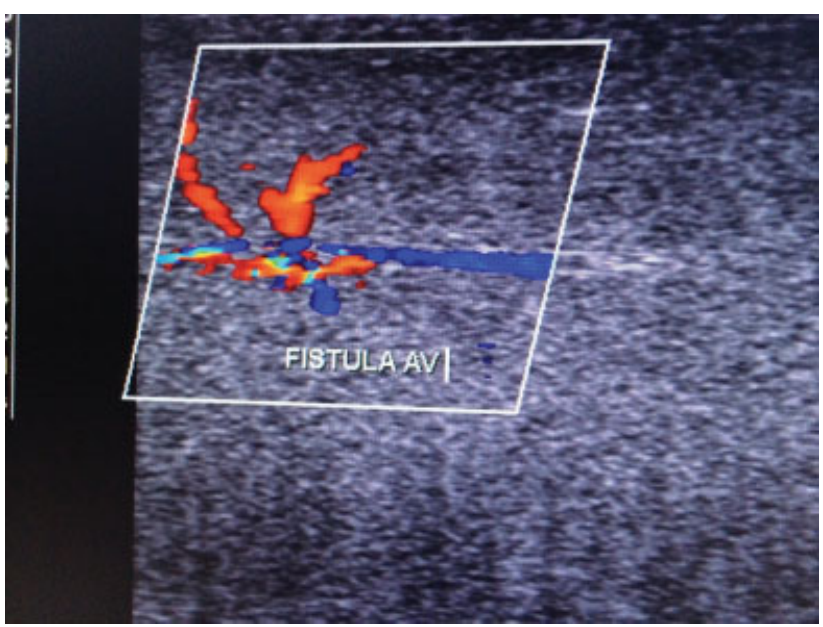

Fig. 11 Fístula arteriovenosa en el cuerpo cavernoso izquierdo en ecografía doppler de pene.

sexual prolongada, infecciones o tumores pelvianos, uso de anillos constrictores en el pene, ciertas toxicomanías, estados de trombosis venosa profunda de miembros inferiores, estados de hipercoagulabilidad, sobredistensión vesical, y poscirugía de hernia inguinal. La ecografía doppler de pene podría corroborar el diagnóstico. El manejo suele ser médico, similar al propuesto para las flebectasias (-Figura 10). ${ }^{22-26}$

\section{Fistulas Arteriovenosas}

Las fistulas arteriovenosas del pene también suelen ser un misterio. Pueden originarse por una malformación congénita o como una manifestación, incluso tardía, de un trauma genital que puede ser coital o producido por agujas en tratamientos de autinyección de vasoactivos. El mayor problema derivado de las fístulas arteriovenosas peneanas es su capacidad de producir priapismo de alto flujo, llamado también no isquémico, en pacientes de todas las edades. La ecografía doppler de pene puede detectar áreas focales de turbulencia con una sensibilidad cercana al $100 \%$, pero este método necesita ser complementado con la arteriografía, para que se pueda realizar el tratamiento definitivo, que suele ser endovascular por radiología intervencionista, con angiografía de la pudenda y embolización selectiva (-Figura 11).$^{27,28}$

\section{Conclusiones}

El pene es objeto de una serie de variaciones anatómicas y de lesiones benignas que pueden alterar su superficie y rara vez tener manifestación clínica, como las venas tortuosas y las flebectasias. Asimismo, hay otro grupo de lesiones, estas sí patológicas, que pueden afectar la integridad del órgano, interferir en su función, y provocar complicaciones mayores, incluyendo las fístulas arteriovenosas peneanas y las fugas venosas dorsales. Otras alteraciones pueden afectar severamente la estética del pene, tal como las malformaciones venosas del glande, o alarmar a pacientes y terapeutas por su presentación aguda y ruidosa, tal como la enfermedad de Mondor.

\section{Conflicto de Intereses}

Los autores no tienen conflicto de intereses que declarar.

\section{Referencias}

1 Uribe JF, Gaviria JA. Breve atlas de anatomía externa normal del pene. urol.colomb. Vol. XVII, No. 3: pp 57-66,2008

2 Yura E, Flury S. Cutaneous Lesions of the External Genitalia. Med Clin North Am 2018;102(02):279-300

3 Rothman CP. Case report: varicocele or cirsocele. J Urol 1980;124 (04):566-567

4 http://www.portalesmedicos.com/diccionario_medico/index.php/ Cirsocele. Consultado 11 de septiembre de 2020

5 Balato N, Montesano M, Lembo G. Acquired phlebectasia of the glans penis. J Am Acad Dermatol 1985;13(5 Pt 1):824-826

6 Song D, Wu C, Guo L, Wang L, Li J, Zhang X. Efficacy and safety of DSA-guided percutaneous sclerotherapy for venous malformations of penile region in children. J Pediatr Surg 2021; 56(03):601-604

7 Biagioli M, Sbano P, Taddeucci P, Risulo M, Fimiani M. Sclerotherapy for penile venous malformations. Dermatol Surg 2006;32(07):955-957, discussion 957

8 Uribe JF. Fuga venosa dorsal profunda como causa de disfunción eréctil primaria y secundaria no estándar (serie de 57 casos). Urología Colombiana 2017;26(01):34-40

9 Hsu GL, Hung YP, Tsai MH, et al. Penile veins are the principal component in erectile rigidity: a study of penile venous stripping on defrosted human cadavers. J Androl 2012;33(06):1176-1185

10 Shafik A, Shafik I, El Sibai O, Shafik AA. On the pathogenesis of penile venous leakage: role of the tunica albuginea. BMC Urol 2007Sep 5;7:14. Doi: 10.1186/1471-2490-7-14

11 Berookhim BM. Doppler Duplex Ultrasonography of the Penis. J Sex Med 2016;13(04):726-731

12 Lue TF, Hricak H, Marich KW, Tanagho EA. Vasculogenic impotence evaluated by high-resolution ultrasonography and pulsed Doppler spectrum analysis. Radiology 1985;155(03): 777-781

13 Roy C, Saussine C, Tuchmann C, Castel E, Lang H, Jacqmin D. Duplex Doppler sonography of the flaccid penis: potential role in the evaluation of impotence. J Clin Ultrasound 2000;28(06): 290-294

14 Speel TG, van Langen H, Wijkstra H, Meuleman EJ. Penile duplex pharmaco-ultrasonography revisited: revalidation of the parameters of the cavernous arterial response. J Urol 2003;169 (01):216-220

15 Altinkilic B, Hauck EW, Weidner W. Evaluation of penile perfusion by color-coded duplex sonography in the management of erectile dysfunction. World J Urol 2004;22(05):361-364

16 Benson CB, Vickers MA. Sexual impotence caused by vascular disease: diagnosis with duplex sonography. AJR Am J Roentgenol 1989;153(06):1149-1153

17 Broderick G. Valoración vascular de la disfunción eréctil. En: Lue, Tom. Atlas de la disfunción sexual masculina. San Francisco: Current Medical Group; 2008

18 Hallerstrom M, von Stempel CB, Raheem A, Walkden M. Abnormal deep dorsal vein resulting in veno-occlusive erectile dysfunction. BMJ Case Rep 2018 Jan 18;2018:bcr2017223496. Doi: 10.1136/ bcr-2017-223496

19 Virag R, Paul JF. New classification of anomalous venous drainage using caverno-computed tomography in men with erectile dysfunction. J Sex Med 2011;8(05):1439-1444

20 Kolte S, Ingle P, Kolte M, Bhuyar S. Thulium laser coagulation for venous malformations of glans penis. Asian J Urol 2016;3(02): $110-111$ 
21 López V, Ricart JM, López I, Martín JM, Marton D, Ortega C, Costa S Vascular malformation of the glans penis successfully treated with Nd:YAG laser. Dermatol Online J 2012 Oct 15;18(10):16

22 Braun-Falco 0 . [Clinical manifestations, histology and pathogenesis of the cordlike superficial phlebitis forms]. Dermatol Wochenschr 1955;132(27):705-715

23 Wild J, Wilson L, Bajaj M. Penile Mondor's disease- an understated entity. Urol Case Rep 2020 Apr 2;31:101176. Doi: 10.1016/j. eucr.2020.101176

24 Mukendi AM, Mahlobo F. Penile Mondor's disease: Clinical and sonographic images. Clin Case Rep 2019;7(11):2283-2284

25 Kostrzewa O, Kołodziej P, Majdan M, Zwolak R, Górak A, Bar K. [Mondor's disease or systemic sclerosis - diagnostic and therapeutic dilemmas]. Wiad Lek 2018;71(1 pt 1):93-95
26 Contreras P, Stoisa D, Pfíster M, Florenzano N, Torsiglieri L, Mazza O. Trombosis de la vena dorsal del pene (flebitis peneana de Mondor): diagnóstico clínico y ecográfico. SAUMB Ultrasonido 2003;2(03):211-215

27 Fiore CM, Pellegrino ML, Trivedi MC. Chronic recurrent priapism: A high flow state secondary to an arteriovenous fistula of the corpus cavernosum. Urol Case Rep 2019 Nov 1;28:101068. Doi: 10.1016/j.eucr.2019.101068

28 Parascani R, Palleschi G, Bova G, et al. Arteriovenous intracavernous post-traumatic fistula: clinical management and treatment by superselective embolization. Urology 2004;63(02): $380-382$ 